\title{
MODULAR REPRESENTATIONS OF LOEWY LENGTH TWO
}

\author{
M. E. CHARKANI and S. BOUHAMIDI
}

Received 23 October 2002

\begin{abstract}
Let $G$ be a finite $p$-group, $K$ a field of characteristic $p$, and $J$ the radical of the group algebra $K[G]$. We study modular representations using some new results of the theory of extensions of modules. More precisely, we describe the $K[G]$-modules $M$ such that $J^{2} M=0$ and give some properties and isomorphism invariants which allow us to compute the number of isomorphism classes of $K[G]$-modules $M$ such that $\operatorname{dim}_{K}(M)=\mu(M)+1$, where $\mu(M)$ is the minimum number of generators of the $K[G]$-module $M$. We also compute the number of isomorphism classes of indecomposable $K[G]$-modules $M$ such that $\operatorname{dim}_{K}(\operatorname{Rad}(M))=1$.
\end{abstract}

2000 Mathematics Subject Classification: 16G10, $20 \mathrm{C} 05$.

1. Introduction. Let $K$ be a field of characteristic $p>0$, and $G$ a finite $p$ group. The aim of this paper is to study $K[G]$-modules of Loewy length two; these are $K[G]$-modules $M$ such that $J^{2} M=0$, where $J$ is the radical of the group algebra $K[G]$ (see [8]). Another characterization is the following: a $K[G]$ module is of Loewy length two if and only if its annihilator $I$ is not a maximal ideal containing $J^{2}$. In general, the Loewy length of a $K[G]$-module $M$ is the smallest integer $n$ such that $J^{n} \cdot M=0$; observe that $n$ exists because $K[G]$ is artinian, and $J$ is a nilpotent ideal. The Loewy length is equal to the length of the kernel filtration of $M$ (see [5]), defined by

$$
M^{(n)}=\left\{x \in M \mid J^{n} \cdot x=0\right\} .
$$

Modules of Loewy length $n$ are described in Propositions 2.1 and 2.2; as a consequence, we find that every $K[G]$-module of Loewy length two is an extension of $K^{s}$ by $K^{t}$, where $s, t$ are positive integers, and $G$ acts trivially on $K^{s}$ and $K^{t}$.

Since the Krull-Schmidt-Azumaya theorem holds for $K[G]$-modules, it is more convenient to study indecomposable $K[G]$-modules. In Proposition 3.1 and Corollary 3.2, we give necessary conditions for a $K[G]$-module of Loewy length two to be indecomposable. If $M$ is indecomposable of Loewy length two, then $J \cdot M=M^{G}$, the subspace consisting of elements of $M$ invariant under the $G$-action (see Theorem 3.4). In Proposition 3.7 we will see that this result is no longer valid if we consider modules of higher Loewy length. As another consequence of Theorem 3.4, we find that the minimum number of generators of an indecomposable $K[G]$-module $M$ of Loewy length two, extension of $K^{s}$ by $K^{t}$, is equal to $s$; in particular, if $s=1$, then $M$ is cyclic and $\operatorname{dim}_{K}\left(M^{G}\right)=t$. 
Propositions 4.1 and 4.2 give a classification of indecomposable $K[G]$-modules of Loewy length two. In Theorem 5.5, we determine the number $\eta(s, m)$ of isomorphism classes of indecomposable $K[G]$-modules $M$ of dimension $s+1$ and of Loewy length two such that $\operatorname{dim}_{K}(\operatorname{Rad}(M))=1$, and $m=\mu(G)$ is the minimum number of generators of $G$. We distinguish two different cases: if $s>\mu(G)$, then all modules are decomposable, and if $s \leq \mu(G)$, then the number of isomorphism classes of indecomposable modules is equal to the number of $s$-dimensional subspaces of $K^{m}$.

Throughout this paper, $K$ will be a commutative field of characteristic $p>0$, $G$ a finite $p$-group, and $K[G]$ the group algebra. We denote by $J$ the Jacobson radical of $K[G]$ and $\mu(G)$ the minimum number of generators of $G$. A $K[G]$-module $M$ will be assumed to be finitely generated; $\mu(M)$ is the minimal number of generators of $M$, and we will denote

$$
\begin{aligned}
M^{G} & =\{m \in M \mid g \cdot m=m \forall g \in G\}, \\
\operatorname{Inv}_{G} M & =\{g \in G \mid g \cdot m=m \forall m \in M\} .
\end{aligned}
$$

Also recall that the sequence $\left(J^{k}\right)_{k}$ is called the principal filtration of $K[G]$. The descending chain $\left(J^{k} M\right)_{k}$ of submodules of $M$ is called the Loewy sequence of $M$ (see [8, page 284]). The sequence $\left(J^{k} M\right)_{k}$ is also called the $J$-filtration of $M$.

2. Preliminaries on extensions of modules. Let $\Lambda$ be a commutative ring and $L$ and $N$ two $\Lambda$-modules. We recall (see $[1,6,10]$ ) that an extension of $L$ by $N$ is a triple $(f, M, g)$, where $M$ is a $\Lambda$-module and $f, g$ are $\Lambda$-module homomorphisms such that the sequence

$$
0 \longrightarrow N \stackrel{f}{\longrightarrow} M \stackrel{g}{\longrightarrow} L \longrightarrow 0
$$

is exact. Two extensions $(f, M, g)$ and $\left(f^{\prime}, M^{\prime}, g^{\prime}\right)$ of $L$ by $N$ are equivalent if there exists an isomorphism of $\Lambda$-modules $\phi: M \rightarrow M^{\prime}$ such that $\phi \circ f=f^{\prime}$ and $g^{\prime} \circ \phi=g$. Let $\bar{E}_{\Lambda}(L, N)$ be the set of equivalence classes of extensions of $L$ by $N$. If $\Lambda=K[G]$, then it is well known (see [9], [3, Section 75], and [4, Proposition 25.10]) that

$$
\bar{E}_{\Lambda}(L, N) \cong H^{1}(G, T),
$$

where $T=\operatorname{Hom}_{K}(L, N)$ is a $K[G]$-module, by taking $h^{\sigma}=\sigma \circ h \circ \sigma^{-1}$ for all $h \in T$ and $\sigma \in G$.

For a cocycle $\alpha \in Z^{1}(G, T)$, the corresponding extension is $M=N \times{ }_{\alpha} L$, which is equal to $N \times L$ as a vector space, with $G$-action

$$
g \cdot(m, n)=(g m+\alpha(g)(g n), g n) .
$$


Proposition 2.1. Let $n$ be a positive integer. Any $K[G]$-module $M$ of Loewy length $n$ is isomorphic to $K^{t} \times_{\alpha} L$, where $t=\operatorname{dim}_{K}\left(J^{n-1} M\right), L$ is a $K[G]$-module of Loewy length $n-1$, and $\alpha \in Z^{1}(G, T)$, with $T=\operatorname{Hom}_{K}\left(L, K^{t}\right)$.

Proof. The module $J^{n-1} M$ is a $K[G]$-module of Loewy length 1 and is isomorphic to $K^{t}$, where $t$ is a positive integer. The module $M$ is an extension of $L=M / J^{n-1} M$ which is a $K[G]$-module of Loewy length $n-1$ by $J^{n-1} M$ which is a trivial $K[G]$-module.

Proposition 2.2. Let $n$ be a positive integer. Any $K[G]$-module of Loewy length $n$ is isomorphic to $N \times_{\alpha} K^{s}$, where $s=\mu(M), N$ is a $K[G]$-module of Loewy length $n-1$, and $\alpha \in Z^{1}(G, T)$, with $T=\operatorname{Hom}_{K}\left(K^{s}, N\right)$.

Proof. The module $M$ is an extension of $M / J M$ by $J M$, and it is clear that $J M$ is a $K[G]$-module of Loewy length $n-1$ and $M / J M$ is a trivial $K[G]$-module.

Recall from [11] that $H^{1}(G, T)=\operatorname{Hom}(G, T)$ if $T=\operatorname{Hom}_{K}\left(K^{s}, K^{t}\right)$.

COROLLARY 2.3. A $K[G]$-module of Loewy length two is isomorphic to $K^{t} \times_{\alpha}$ $K^{s}$, where $0 \neq \alpha \in \operatorname{Hom}(G, T)$ and $T=\operatorname{Hom}_{K}\left(K^{s}, K^{t}\right)$.

Let $M=N \times{ }_{\alpha} L$ be a $K[G]$-module and consider the $K[G]$-modules

$$
\operatorname{Ker}_{G}(\alpha)=\bigcap_{\sigma \in G} \operatorname{Ker}(\alpha(\sigma)), \quad \operatorname{Im}_{G}(\alpha)=\sum_{\sigma \in G} \alpha(\sigma)(L)
$$

We put $\operatorname{Rg}(\alpha)=\operatorname{dim}_{K}\left(\operatorname{Im}_{G}(\alpha)\right)$. Let $\left\{\sigma_{1}, \sigma_{2}, \ldots, \sigma_{m}\right\}$ be a system of generators of $G$, where $m=\mu(G)$ is the minimal number of generators of $G$, and let $\left(e_{1}, \ldots, e_{s}\right)$ be a basis of $L$. We define $\alpha_{j}\left(\sigma_{i}\right)=\alpha\left(\sigma_{i}\right)\left(e_{j}\right)$. It is then easy to see that $\left\{\alpha_{j}\left(\sigma_{i}\right) \mid i \in\{1, \ldots, m\}, j \in\{1, \ldots, s\}\right\}$ is a system of generators of $\operatorname{Im}_{G}(\alpha)$. Therefore,

$$
\operatorname{Rg}(\alpha) \leq \operatorname{Inf}\left(\operatorname{dim}_{K}(N), \mu(G) \operatorname{dim}_{K}(L)\right)
$$

For a $K[G]$-module $M$, we define

$$
\operatorname{Inv}_{G}(M)=\{\sigma \in G \mid \sigma \cdot x=x \forall x \in M\}
$$

Proposition 2.4. Let $M=N \times{ }_{\alpha} K^{s}$ be a $K[G]$-module extension of $K^{s}$ by $N$. If $H$ is a subgroup of $\operatorname{Inv}_{G}(N)$, then

$$
M^{H}=N \times_{\alpha} \operatorname{Ker}_{H}(\alpha) .
$$

Proof. Take $(m, n) \in M^{H}$. For every $h \in H$, we have that $h \cdot(m, n)=$ $(m, n)$. But $h \cdot(m, n)=(m+\alpha(h)(h \cdot n), h \cdot n)$, so $h \cdot n=n$ and $\alpha(h)(n)=0$. So $n \in \operatorname{Ker}_{H}(\alpha)$, and therefore $M^{H} \subseteq N \times_{\alpha}\left(\operatorname{Ker}_{H}(\alpha)\right)$. The converse inclusion is easy. 
3. Decomposability of modules of Loewy length two. In this section, we establish some characterizations of the decomposability of $K[G]$-modules of Loewy length two.

Proposition 3.1. Let $M=N \times_{\alpha} L$ be a $K[G]$-module and $\alpha \in Z^{1}(G, T)$.

(1) Each direct summand of $L$ included in $\operatorname{Ker}_{G}(\alpha)$ is a direct summand of $M$.

(2) If $\operatorname{Im}_{G}(\alpha)$ is a direct summand of $N$, then $\operatorname{Im}_{G}(\alpha) \times_{\alpha} L$ is a direct summand of $M$.

Proof. (1) If $L=L^{\prime} \oplus L^{\prime \prime}$, where $L^{\prime}$ and $L^{\prime \prime}$ are two $K[G]$-submodules of $L$ such that $L^{\prime} \subseteq \operatorname{Ker}_{G}(\alpha)$, then $M=N \times{ }_{\alpha} L^{\prime \prime} \oplus 0 \times{ }_{\alpha} L^{\prime}$.

(2) If there exists a $K[G]$-submodule $N^{\prime}$ of $N$ such that $N=\operatorname{Im}_{G}(\alpha) \oplus N^{\prime}$, then $M=N^{\prime} \times{ }_{\alpha} 0 \oplus \operatorname{Im}_{G}(\alpha) \times_{\alpha} L$.

COROLLARY 3.2. If $M=K^{t} \times_{\alpha} K^{s}$ is an indecomposable $K[G]$-module of Loewy length two, then $\operatorname{Rg}(\alpha)=t$ and $\operatorname{Ker}_{G}(\alpha)=\{0\}$.

Proof. This is an immediate consequence of Proposition 3.1 and the fact that $\operatorname{Rg}(\alpha)=\operatorname{dim}_{K}\left(\operatorname{Im}_{G}(\alpha)\right)$.

REMARK 3.3. We deduce from Corollary 3.2 that $M=K^{t} \times_{\alpha} K^{s}$ is a decomposable $K[G]$-module if $s \mu(G)<t$.

THEOREM 3.4. Let $G$ be a finite $p$-group. If $M$ is an indecomposable $K[G]$ module of Loewy length two, then $M^{G}=J M$.

Proof. We can write $M=J M \times{ }_{\alpha} K^{s}$ and, by Proposition 2.4, we have $M^{G} \simeq$ $J M \times \operatorname{Ker}_{G}(\alpha)$. The module $M$ is an indecomposable $K[G]$-module, so it follows from Corollary 3.2 that $\operatorname{Ker}_{G}(\alpha)=\{0\}$. Therefore, $\operatorname{dim}_{K}\left(M^{G}\right)=\operatorname{dim}_{K}(J M)$ and $M^{G}=J M$ since $J M \subseteq M^{G}$.

COROLLARY 3.5. Let $G$ be a finite $p$-group. Let $M$ be a $K[G]$-module of Loewy length two such that $\operatorname{dim}_{K}(\operatorname{Rad}(M))=1$. Then $M$ is an indecomposable $K[G]$ module if and only if $M^{G}=J M$.

Proof. It suffices to prove one direction. The ring $K[G]$ is a local ring (see [8]), so $\operatorname{Rad}(M)=J M$. But $\operatorname{dim}_{K}(\operatorname{Rad}(M))=1$ and $M^{G}=J M$, so $\operatorname{dim}_{K}\left(M^{G}\right)=1$, and therefore $M$ is an indecomposable $K[G]$-module.

COROLLARY 3.6. If $M$ is an indecomposable $K[G]$-module of Loewy length two, then $M$ is isomorphic to $K^{t} \times_{\alpha} K^{s}$ with $t=\operatorname{dim}_{K}\left(M^{G}\right)$ and $s=\mu(M)$.

PROof. This is a consequence of Theorem 3.4 and the fact that $K[G]$ is a local ring, and therefore $\mu(M)=\operatorname{dim}_{K}(M / J M)$.

Let $M$ be an indecomposable $K[G]$-module of Loewy length $n>2$. One might conjecture that $J^{n-1} M \subseteq M^{G}$. In Proposition 3.7, we will show that the inclusion $J^{n-1} M \subset M^{G}$ may be strict. 
Proposition 3.7. Let $G$ be a finite $p$-group, with $p$ an odd prime and $n$ an integer such that $\operatorname{dim}_{K}\left(J^{n-2} / J^{n-1}\right) \geq 2$. Then there exists an indecomposable cyclic $K[G]$-module $M$ of Loewy length $n$ such that $J^{n-1} M$ is strictly included in $M^{G}$.

Proof. The ring $K[G]$ being an artinian and noetherian, $J$ has a composition series. Hence, there exists an ideal $I$ of $K[G]$ such that $J^{n-1} \subset I \subset J^{n-2}$ and such that $J^{n-2} / I$ is an irreducible $K[G]$-module (isomorphic to $K$ ). The ideal $I J$ is strictly included in $J^{n-1}$; otherwise $J^{n-2}$ is included in $I$, and therefore $J^{n-2}=I$. So, $J^{n} \subset I J \subset J^{n-1}$ and then the Loewy length of the $K[G]$ module $K[G] / I J$ is $n$. We have $M^{G}=(I J: J) / I J$. Since $J^{n-1} \subset I \subseteq(I J: J)$ and $J^{n-1} M=J^{n-1} / I J, J^{n-1} M$ is strictly included in $M^{G}$.

4. Isomorphism classes of modules of Loewy length two. When we study the modules of Loewy length two, we need to know when two modules $M=$ $K^{t} \times_{\alpha} K^{s}$ and $M^{\prime}=K^{t} \times_{\beta} K^{s}$ are isomorphic. This is what we do in the next proposition.

Proposition 4.1. Let $M=K^{t} \times_{\alpha} K^{s}$ and $M^{\prime}=K^{t} \times_{\beta} K^{s}$ be indecomposable modules of Loewy length two. Then $M$ and $M^{\prime}$ are isomorphic if and only if there exist an automorphism $\varphi$ of $K^{t}$ and an automorphism $\psi$ of $K^{s}$ such that $\varphi \cdot \alpha \cdot \psi=\beta$, where $(\varphi \cdot \alpha \cdot \psi)(g)=\varphi \circ \alpha(g) \circ \psi$.

Proof. The proof is an adaption of the proof of [3, Lemma 81.8]: if $M$ and $M^{\prime}$ are indecomposable, then, by Theorem $3.4, M^{G}=M^{\prime G}=K^{t} \times\{0\}$, and therefore any homomorphism from $M$ to $M^{\prime}$ is a triangular matrix of homomorphisms and keeps $K^{t}$ globally invariant. For more details, the reader may consult [2].

Proposition 4.2. Let $M=K^{t} \times_{\alpha} K^{s}$ and $M^{\prime}=K^{t} \times_{\beta} K^{s}$ be two indecomposable extensions of $K^{s}$ by $K^{t}$. If $M$ and $M^{\prime}$ are isomorphic, then $\operatorname{Rg}(\alpha)=\operatorname{Rg}(\beta)$.

Proof. If $M$ and $M^{\prime}$ are isomorphic, then there exist automorphisms $\varphi$ and $\psi$ of $K^{t}$ and $K^{s}$ such that $\varphi \cdot \alpha \cdot \psi=\beta$. Then $\operatorname{Im}_{G}(\beta)$ is generated by $\beta\left(\sigma_{j}\right)\left(e_{i}\right)$, and therefore $\operatorname{dim}_{K}\left(\operatorname{Im}_{G}(\beta)\right)=\operatorname{dim}_{K} \alpha\left(\sigma_{j}\right)\left(\psi\left(e_{i}\right)\right)=\operatorname{dim}_{K}\left(\operatorname{Im}_{G}(\alpha)\right)$.

The converse of Proposition 4.2 is not true in general; under a certain hypothesis, we have equivalence.

Proposition 4.3. Let $G$ be a finite $p$-group and $s, t$ two integers such that $t=s \mu(G)$. Let $M=K^{t} \times{ }_{\alpha} K^{s}$ and $M^{\prime}=K^{t} \times{ }_{\beta} K^{s}$ be two indecomposable extensions of $K^{s}$ by $K^{t}$. Then $M \simeq M^{\prime}$ if and only if $\operatorname{Rg}(\alpha)=\operatorname{Rg}(\beta)$.

PROOF. If $M=K^{t} \times_{\alpha} K^{s}$ and $M^{\prime}=K^{t} \times_{\beta} K^{s}$ are indecomposable such that $\operatorname{Rg}(\alpha)=\operatorname{Rg}(\beta)$, then $\operatorname{Rg}(\alpha)=\operatorname{Rg}(\beta)=t=s m$. The system $\left(\alpha\left(\sigma_{j}\right) e_{i}\right)$ has $t$ elements and generates $K^{t}$, so it is a basis of $K^{t}$. Similarly, $\left(\beta\left(\sigma_{j}\right) e_{i}\right)$ is a basis of $K^{t}$. Then we define an automorphism $\varphi$ of $K^{t}$ by $\varphi\left(\alpha\left(\sigma_{j}\right)\left(e_{i}\right)\right)=\beta\left(\sigma_{j}\right)\left(e_{i}\right)$. The desired conclusion is deduced from Proposition 4.1 . 
COROLlARY 4.4. Let $G$ be a finite $p$-group such that $m=\mu(G)$.

(1) There exists only one isomorphism class of cyclic $K[G]$-modules $M$ of Loewy length two such that $\operatorname{dim}_{K}(M)=m+1$.

(2) If $G=(\mathbb{Z} / p \mathbb{Z})^{m}$, then any cyclic $K[G]$-module $M$ of Loewy length two such that $\operatorname{dim}_{K}(M)=m+1$ is isomorphic to $K[G] / J^{2}$.

Proof. (1) Any cyclic $K[G]$-module $M$ is indecomposable. Then, by Corollary 3.6, a cyclic $K[G]$-module $M$ of Loewy length two is isomorphic to $K^{t} \times{ }_{\alpha} K$ with $t=\operatorname{dim}_{K}\left(M^{G}\right)$. Since $\operatorname{dim}_{K}(M)=m+1, t=m$ and all such modules are isomorphic to $K^{m} \times{ }_{\alpha} K$.

(2) If $M$ is a cyclic $K[G]$-module such that $\operatorname{dim}_{K}(M)=m+1$, then $M$ is indecomposable and isomorphic to $K[G] / I$, where $I$ is a proper ideal of $K[G]$ containing $J^{2}$ since $\operatorname{dim}_{K}\left(K[G] / J^{2}\right)=m+1$ (see [8, page 119, Theorem 2.3]), and then $I=J^{2}$.

REMARK 4.5. Proposition 4.3 asserts that if $s$ and $t$ are two integers such that $t=s \mu(G)$, then there exists only one isomorphism class of indecomposable $K[G]$-modules $M$ of Loewy length two such that $\operatorname{dim}_{K}\left(M^{G}\right)=t$ and $\mu(M)=s$.

5. Extensions of $K^{s}$ by $K$. We have seen in Corollary 3.6 that all indecomposable modules of a Loewy length two extension of $K$ by $K^{s}$ are cyclic, and therefore they are isomorphic if and only if they have the same annihilator. In this section, we will study the isomorphism classes of modules of a Loewy length two extension of $K^{s}$ by $K$, with $s$ being a nonzero integer.

Proposition 5.1. Let $M=K \times_{\alpha} K^{s}$ be a $K[G]$-module extension of $K^{s}$ by $K$. Then $M$ is an indecomposable $K[G]$-module if and only if $\operatorname{Ker}_{G}(\alpha)=\{0\}$.

Proof. If $M=K \times_{\alpha} K^{s}$ and $\operatorname{Ker}_{G}(\alpha)=\{0\}$, then we conclude from Proposition 2.4 that $\operatorname{dim}_{K}\left(M^{G}\right)=1$, and therefore $M$ is an indecomposable $K[G]$ module.

From now on, we assume that $K$ is the finite field of $q=p^{r}$ elements. From [7], we recall the following lemma.

LEMMA 5.2. Let $V$ be a $K$-vector space of dimension $n$. The number of $m$ dimensional subspaces of $V$ is given by the formula

$$
\left[\begin{array}{c}
n \\
m
\end{array}\right]=\frac{\prod_{i=0}^{m-1}\left(q^{n}-q^{i}\right)}{\prod_{i=0}^{m-1}\left(q^{m}-q^{i}\right)} .
$$

Proposition 5.3. Let $s$ and $m$ be nonzero integers. The number of orbits of the left action of $\operatorname{Aut}\left(K^{s}\right)$ on $\operatorname{Hom}_{K}\left(K^{m}, K^{s}\right)$ is equal to

$$
\sum_{k=0}^{\min (m, s)}\left[\begin{array}{l}
m \\
k
\end{array}\right] .
$$


Proof. Consider two homomorphisms $f, g: K^{m} \rightarrow K^{s}$. Then $f$ and $g$ are in the same orbit if and only if there exists an automorphism $\varphi$ of $K^{s}$ such that $\varphi \circ f=g$. Now $\varphi \circ f=g$ if and only if $\operatorname{Ker} f=\operatorname{Ker} g$, so two homomorphisms are in the same orbit if and only if they have the same kernel. Therefore, the number of orbits of the left action of $\operatorname{Aut}\left(K^{s}\right)$ on $\operatorname{Hom}_{K}\left(K^{m}, K^{s}\right)$ is equal to the number of subspaces of $K^{m}$ of dimension $k$, where $k$ is an integer such that $m-\min (m, s) \leq k \leq m$. Now

$$
\left[\begin{array}{c}
m \\
k
\end{array}\right]=\left[\begin{array}{c}
m \\
m-k
\end{array}\right]
$$

hence,

$$
\sum_{k=m-\min (m, s)}^{m}\left[\begin{array}{c}
m \\
k
\end{array}\right]=\sum_{k=0}^{\min (m, s)}\left[\begin{array}{c}
m \\
k
\end{array}\right] .
$$

LEMMA 5.4. If $M=K \times_{\alpha} K^{s}$ is a $K[G]$-module of Loewy length two, then $J \cdot M=K \times\{0\}$.

Proof. Let $M=K \times_{\alpha} K^{s}$ be a $K[G]$-module of Loewy length two. The projection map $\mathrm{pr}_{2}$ is a $K[G]$-homomorphism from $K \times_{\alpha} K^{s}$ to $K^{s}$ so $\operatorname{pr}_{2}\left(J \cdot\left(K \times_{\alpha}\right.\right.$ $\left.\left.K^{s}\right)\right) \subseteq J \cdot K^{s}$ (see [8]). Since $K^{s}$ is a completely reducible $K[G]$-module, we have $J \cdot K^{s}=0$, and therefore $\operatorname{pr}_{2}(J \cdot M)=0$. We have $J \cdot M \subseteq K \times \operatorname{Ker}_{G}(\alpha)$ because $M$ is a $K[G]$-module of Loewy length two. It follows that $J \cdot M \subseteq M^{G}$ and $M^{G}=K \times \operatorname{Ker}_{G}(\alpha)$ (see Propositions 2.4 and 3.1). Since $\operatorname{pr}_{2} J \cdot M=0$, we have $J \cdot M=K \times\{0\}$ or $J \cdot M=0$. If $J \cdot M=0$, then $M$ is a completely reducible $K[G]$-module, hence a $K[G]$-module of Loewy length one, which contradicts our hypothesis that $M$ is of Loewy length two. We conclude that $J \cdot M=K \times\{0\}$.

Let $G$ be a finite abelian $p$-group with $\mu(G)=m(\mu(G)$ is the minimal number of generators of $G)$. Let $\eta(s, m)$ be the number of isomorphism classes of $K[G]$ modules of Loewy length two which are extensions of $K^{s}$ by $K$.

THEOREM 5.5. Let $s$ be a positive integer and let $G$ and $m$ be as above. Then

$$
\eta(s, m)=\sum_{k=1}^{\min (m, s)}\left(\frac{\prod_{i=0}^{k-1}\left(q^{m}-q^{i}\right)}{\prod_{i=0}^{k-1}\left(q^{k}-q^{i}\right)}\right) .
$$

Proof. Let $T=\operatorname{Hom}_{K}\left(K^{s}, K\right)$ and take a basis $\left\{e_{1}, \ldots, e_{s}\right\}$ of $K^{s}$ and a basis $\left\{e_{1}^{\prime}, \ldots, e_{m}^{\prime}\right\}$ of $K^{m}$. Also consider the map

$$
f: Z^{1}(G, T) \longrightarrow \operatorname{Hom}_{K}\left(K^{s}, K^{m}\right), \quad f(\alpha)=\widetilde{\alpha},
$$


where $\tilde{\alpha}(x)=\sum_{i=1}^{m} \alpha\left(\sigma_{i}\right)(x) e_{i}^{\prime}$. If $M=K \times_{\alpha} K^{s}$ is a $K[G]$-module of Loewy length two, then $\widetilde{\alpha} \neq 0$ and $\operatorname{Ker} \widetilde{\alpha}=\operatorname{Ker}_{G}(\alpha)$.

Consider a second $K[G]$-module $M^{\prime}=K \times_{\beta} K^{s}$ of Loewy length two and assume that $\varphi: M \rightarrow M^{\prime}$ is an isomorphism. It follows from Lemma 5.4 that $\varphi(K \times\{0\}) \subseteq(K \times\{0\})\left(\varphi(J \cdot M) \subseteq J \cdot M^{\prime}\right.$, see [8]). Then there exists an automorphism $\psi$ of $K^{s}$ such that

$$
\alpha\left(\sigma_{i}\right) \circ \psi=\beta\left(\sigma_{i}\right)
$$

for all $i \in\{1, \ldots, m\}$, which is equivalent to

$$
\widetilde{\alpha} \circ \psi=\tilde{\beta}
$$

so the number of isomorphism classes of $K[G]$-modules that are extensions of $K^{s}$ by $K$ is equal to the number of orbits of the right action of $\operatorname{Aut}\left(K^{s}\right)$ on $\operatorname{Hom}_{K}\left(K^{s}, K^{m}\right)-\{0\}$, which is equal to the number of orbits of the left action of $\operatorname{Aut}\left(K^{s}\right)$ on $\operatorname{Hom}_{K}\left(K^{m}, K^{s}\right)-\{0\}$, and our result follows from Proposition 5.3.

Let $M$ be a $K[G]$-module of Loewy length $n$, then $J^{n-1} M \subseteq M^{G}$. In order to study the quotient $M^{G} / J^{n-1} M$, we need the following definition.

Definition 5.6. Let $M$ be a $K[G]$-module of Loewy length two. We call $\operatorname{dim}_{K}\left(M^{G} / J M\right)$ the index of $M$.

By Theorem 3.4, the index of an indecomposable $K[G]$-module of Loewy length two is equal to zero.

COROLlary 5.7. Let $n$ be a nonzero integer and $G$ a finite abelian $p$-group, with $\mu(G)=m$. Then the number of isomorphism classes of $K[G]$-modules of Loewy length two, index $r$, minimal number of generators $n$ and dimension $n+1$ is equal to $[\underset{(n-r)}{m}]$.

Proof. It follows from Proposition 2.4 and Theorem 3.4 that such a module is of the form $K \times_{\alpha} K^{n}$, with $r=\operatorname{dim}_{K}\left(M^{G} / J \cdot M\right)=\operatorname{dim}_{K}\left(\operatorname{Ker}_{G}(\alpha)\right)$ and $\operatorname{Ker}_{G}(\alpha)=\operatorname{Ker}(\widetilde{\alpha})$. Therefore, the number of isomorphism classes is equal to the number of orbits of the left action of $\operatorname{Aut}\left(K^{n}\right)$ on the set of homomorphisms $f: K^{m} \rightarrow K^{n}$ such that $\operatorname{dim}_{K}(\operatorname{Im} f)=n-r$ (see Theorem 5.5), which is equal to $\left[\begin{array}{c}m \\ (n-r)\end{array}\right]$, by Proposition 5.3.

COROLlary 5.8. Let $G$ be a finite abelian $p$-group and $s$ and $m$ two positive integers.

(1) If $s \leq m$, then there exist $\left[\begin{array}{c}m \\ s\end{array}\right]$ isomorphism classes of indecomposable $K[G]$-modules which are extensions of $K^{s}$ by $K$.

(2) If $s>m$, then all $K[G]$-modules which are extensions of $K^{s}$ by $K$ are decomposable. 
Proof. If $M$ is an indecomposable $K[G]$-module, then the index of $M$ is equal to zero. The result is therefore an immediate consequence of Corollary 5.7.

REMARK 5.9. If $s=m$, then there exists only one isomorphism class of an indecomposable $K[G]$-module which is an extension of $K^{s}$ by $K$. Indeed, $\left[\begin{array}{c}m \\ m\end{array}\right]=1$. This is a particular case of Proposition 4.3 .

NoTE 5.10. (1) Let $M=N \times_{\alpha} L$ be an $R[G]$-module extension of $L$ by $N$ and let $L^{\prime}$ be a submodule of the $R[G]$-module $L$, and $N^{\prime}$ a submodule of the $R[G]$ module $N$ contained in $\sum_{\sigma \in G} \alpha(\sigma)\left(L^{\prime}\right)$. Then we can consider $\alpha$ as a cocycle in $Z^{1}\left(G, T^{\prime}\right)$, where $T^{\prime}=\operatorname{Hom}_{K}\left(L^{\prime}, N^{\prime}\right)$, and $N^{\prime} \times_{\alpha} L^{\prime}$ is a submodule of the $R[G]$-module $M$.

(2) Let $\mathscr{S}=\left\{\sigma_{1}, \sigma_{2}, \ldots, \sigma_{m}\right\}$ be a system of generators of $G=(\mathbb{Z} / p \mathbb{Z})^{m}$ and consider an $m \times s$ matrix $A=\left(a_{i j}\right) \in \mu_{m, s}(K)$. Let $M$ be the $K$-vector space with basis $\mathscr{B}=\left\{u_{1}, u_{2}, \ldots, u_{s+1}\right\}$. We define the following $G$-action on $M$ :

$$
\sigma_{i} \cdot u_{j}= \begin{cases}u_{j}+a_{i j} u_{s+1}, & \text { if } j \leq s \\ u_{j}, & \text { if } j=s+1\end{cases}
$$

$M$ together with the $K[G]$-module structure (5.9) will be denoted by $K\langle A\rangle$. If $s \leq$ $m$ and $A$ is of rank $s$, then it can be shown that $M=K\langle A\rangle$ is an indecomposable $K[G]$-module of Loewy length two such that $\operatorname{dim}_{K}(M)=s+1$ and $\mu(M)=$ $s$. More precisely, $K\langle A\rangle$ is isomorphic to $K \times{ }_{\alpha} K^{s}$, where $\alpha \in Z^{1}(G, T), T=$ $\operatorname{Hom}_{K}\left(K^{s}, K\right)$, and $\alpha\left(\sigma_{i}\right)\left(e_{j}\right)=a_{i j}$.

(3) Every $K[G]$-module as in Corollary 5.8 is isomorphic to a certain $K\langle A\rangle$.

\section{REFERENCES}

[1] H. Cartan and S. Eilenberg, Homological Algebra, 7th ed., Princeton University Press, New Jersey, 1973.

[2] M. E. Charkani and S. Bouhamidi, Caractérisation des classes d'isomorphismes des extensions semi-scindées sur un anneau de groupe, to appear.

[3] C. Curtis and I. Reiner, Representation Theory of Finite Groups and Associative Algebras, 2nd ed., Interscience, New York, 1966.

[4] _ Methods of Representation Theory. Vol. I, Pure and Applied Mathematics, John Wiley \& Sons, New York, 1981.

[5] D. Duval, Sur la structure galoisienne du groupe des unités d'un corps abélien réel de type ( $p, p)$, J. Number Theory 13 (1981), no. 2, 228-245 (French).

[6] P. J. Hilton and U. Stammbach, A Course in Homological Algebra, Graduate Texts in Mathematics, vol. 4, Springer-Verlag, Berlin, 1971.

[7] G. James, Representations of General Linear Groups, London Mathematical Society Lecture Note Series, vol. 94, Cambridge University Press, Cambridge, 1984.

[8] G. Karpilovsky, The Jacobson Radical of Group Algebras, North-Holland Mathematics Studies, vol. 135, North-Holland Publishing, Amsterdam, 1987.

[9] I. Reiner, A survey of integral representation theory, Bull. Amer. Math. Soc. 76 (1970), 159-227. 
[10] K. Roggenkamp and V. Huber-Dyson, Lattices over Orders. I, Lecture Notes in Mathematics, vol. 115, Springer-Verlag, Berlin, 1970.

[11] E. Weiss, Cohomology of Groups, Pure and Applied Mathematics, vol. 34, Academic Press, New York, 1969.

M. E. Charkani: Department of Mathematics, Faculty of Sciences Dhar-Mahraz, University of Sidi Mohammed Ben Abdellah, BP 1796, Fes, Morocco

E-mail address: mcharkani@excite.com

S. Bouhamidi: Department of Mathematics, Faculty of Sciences Dhar-Mahraz, University of Sidi Mohammed Ben Abdellah, BP 1796, Fes, Morocco

E-mail address: sbouhamidi@hotmai 1 .com 


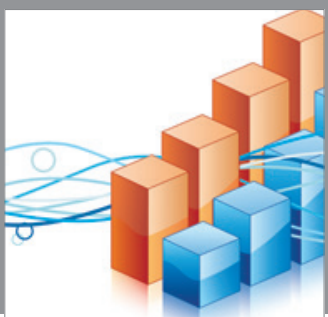

Advances in

Operations Research

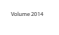

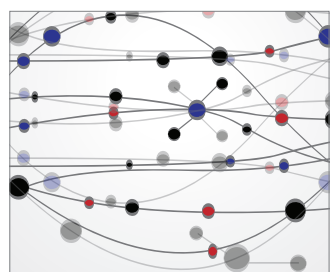

\section{The Scientific} World Journal
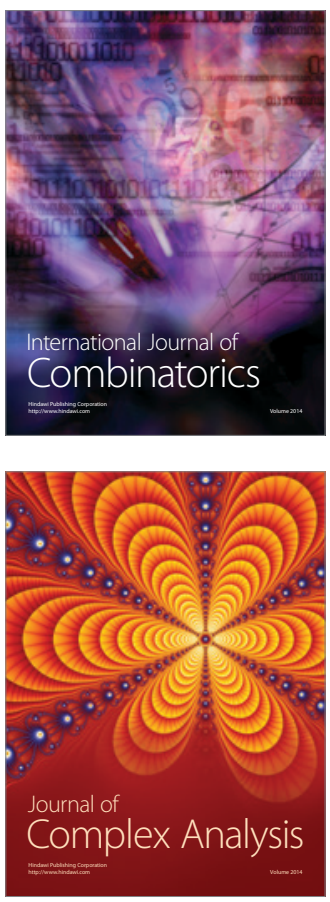

International Journal of

Mathematics and

Mathematical

Sciences
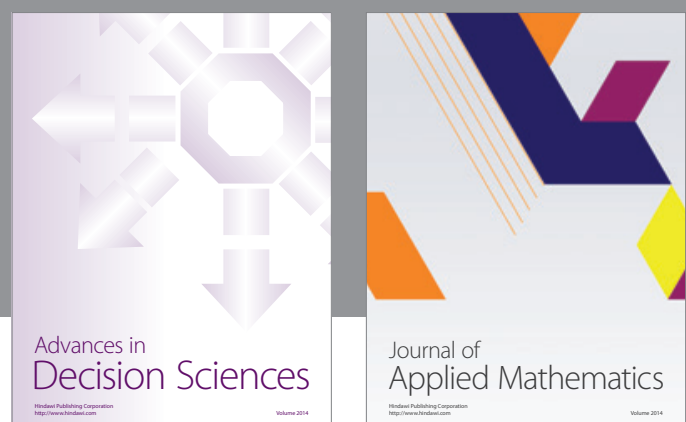

Journal of

Applied Mathematics
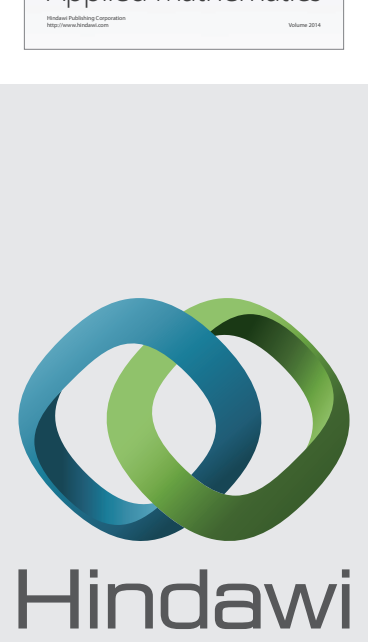

Submit your manuscripts at http://www.hindawi.com
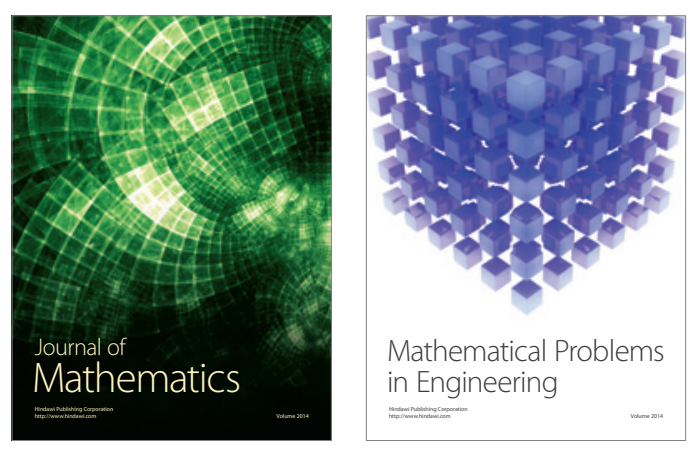

Mathematical Problems in Engineering
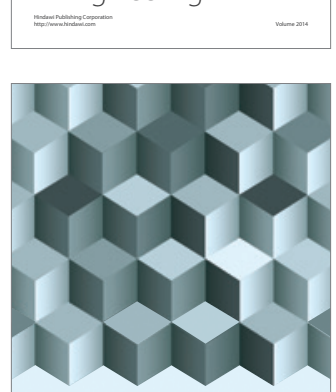

Journal of

Function Spaces
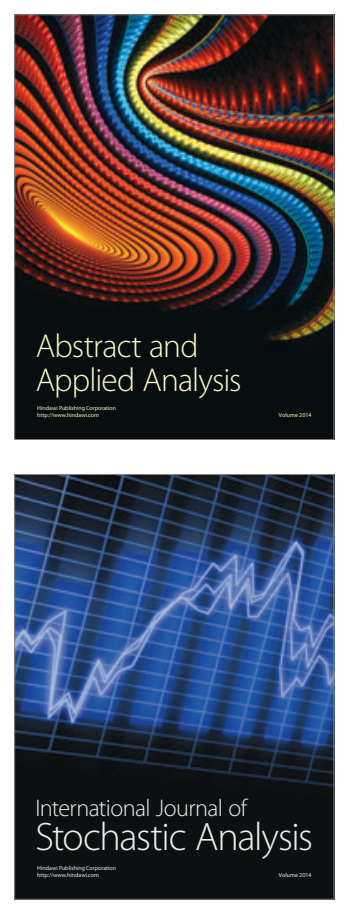

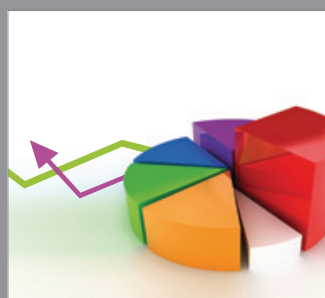

ournal of

Probability and Statistics

Promensencen
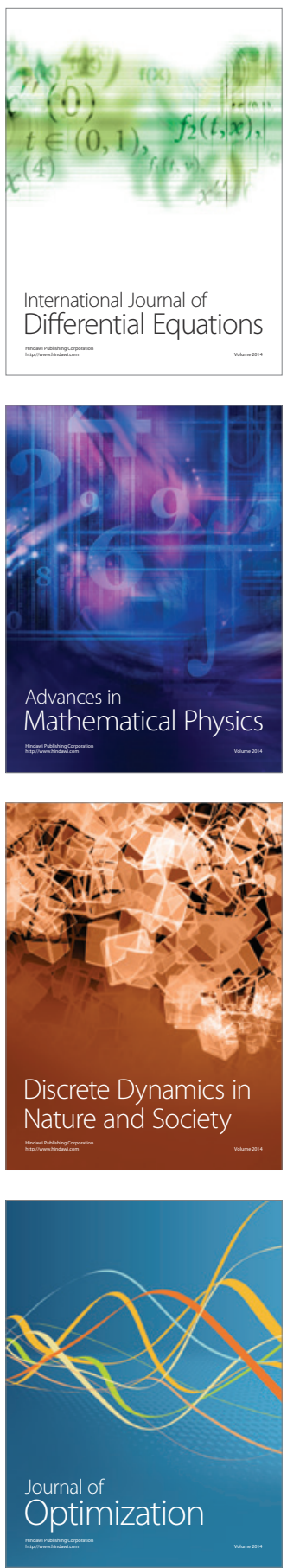